\title{
Aggregation gatekeepers modulate protein homeostasis of aggregating sequences and affect bacterial fitness
}

Jacinte Beerten ${ }^{1,2}$, Wim Jonckheere ${ }^{1,2}$, Stanislav Rudyak ${ }^{1,2}$, Jie Xu $\mathbf{X}^{1,2}$, Hannah Wilkinson ${ }^{1,3}$, Frederik De Smet ${ }^{1,3}$, Joost Schymkowitz ${ }^{1,3,4}$ and Frederic Rousseau ${ }^{1,3,4}$

${ }^{1}$ Switch Laboratory, VIB, University of Leuven, Leuven, Belgium, ${ }^{2}$ Vrije Universiteit Brussel, Pleinlaan 2, B-1050 Brussel and ${ }^{3}$ Switch Laboratory, Department of Cellular and Molecular Medicine, University of Leuven,

B-3000 Leuven, Belgium

${ }^{4}$ To whom correspondence should be addressed.

E-mail: frederic.rousseau@switch.vib-kuleuven.be (F.R.);

joost.schymkowitz@switch.vib-kuleuven.be (J.S.)

Received May 3, 2012; revised May 3, 2012;

accepted May 9, 2012

Edited by Sir Alan Fersht

The most common mechanism by which proteins aggregate consists in the assembly of short hydrophobic primary sequence segments into extended $\beta$-structured agglomerates. A significant enrichment of charged residues is observed at the flank of these aggregation-prone sequence segments, suggesting selective pressure against aggregation. These so-called aggregation gatekeepers act by increasing the intrinsic solubility of aggregating sequences in vitro, but it has been suggested that they could also facilitate chaperone interactions. Here, we address whether aggregation gatekeepers affect bacterial fitness. In Escherichia coli MC4100 we overexpressed GFP fusions with an aggregation-prone segment of $\sigma 32$ (further termed $\sigma 32 \beta$ ) flanked by gatekeeper and nongatekeeper residues and measured pairwise competitive growth. We found that the identity of flanking residues had significant effect on bacterial growth. Overexpression of $\sigma 32 \beta$ flanked by its natural gatekeepers displayed the greatest competitive fitness, followed by other combinations of gatekeepers, while absence of gatekeepers strongly affects bacterial fitness. Further analysis showed the diversity of effects of gatekeepers on the proteostasis of $\sigma 32 \beta$ including synthesis and degradation rates, in vivo aggregation propensity and chaperone response. Our results suggest that gatekeeper residues affect bacterial fitness not only by modulating the intrinsic aggregation propensity of proteins but also by the manner in which they affect the processing of $\sigma 32 \beta-$ GFP by the protein quality control machinery of the cell. In view of these observations, we hypothesize that variation at gatekeeper positions offers a flexible selective strategy to modulate the proteostatic regulation of proteins to the match intrinsic aggregation propensities of proteins with required expression levels.

Keywords: amyloidosis/gatekeeper/protein aggregation/ protein expression/proteostasis

\section{Introduction}

One of the most common mechanisms whereby proteins aggregate consists in the $\beta$-stand association of short aggregation-nucleating sequence segments into insoluble aggregates. Protein misfolding and aggregation generally leads to the suppression of native protein function, and in many cases it also appears to elicit wild-type independent gain-of-function leading to neurodegenerative diseases such as Alzheimer's disease or Parkinson's disease, systemic amyloidoses, cancer and metabolic diseases (Chiti and Dobson, 2006). The fact that most proteins tend to aggregate, even under native conditions, to form amorphous $\beta$-aggregates or cross- $\beta$ amyloid structures shows that $\beta$-aggregation is a generic structural propensity of protein polypeptide chains that is in direct competition with native protein folding (Dobson, 2001; Rousseau et al., 2006a; Fitzpatrick et al., 2011). Indeed, it has been demonstrated that the vast majority of proteins possess at least one and often multiple aggregation-nucleating amino acid segments (Rousseau et al., 2006c; Goldschmidt et al., 2010). Moreover, aggregation propensity and globular structure of proteins are intimately tied as aggregation-prone segments and generally contribute to the hydrophobic core (Linding et al., 2004; De Baets et al., 2011). As a result, although protein folding is a thermodynamically determined process (Anfinsen and Scheraga, 1975), folding efficiency is often challenged by non-native misfolding and aggregation propensities (De Simone et al., 2011).

Given the undissociatable ties of protein aggregation with globular structure and function on the one hand, and on the other hand the detrimental consequences of protein aggregation on the biochemistry of the cell, organisms have evolved control mechanisms to keep aggregation in check (Monsellier and Chiti, 2007; Monsellier et al., 2007; Reumers et al., 2009a; Reumers et al., 2009b; de Groot and Ventura, 2010). One of these strategies includes a sophisticated protein quality control system that consists of molecular chaperones and proteases (Mogk et al., 1999; Guisbert et al., 2004; Balch et al., 2008). These proteins prevent unwanted interactions between unfolded proteins, improve the efficiency of de novo protein folding of newly synthesized proteins, remove misfolded proteins by degradation and resolubilize protein aggregates for subsequent refolding or degradation (Muchowski and Wacker, 2005; Soti et al., 2005). Apart from having developed dedicated protein quality control machinery, selective pressure has also shaped protein sequences in a way to minimize aggregation (Rousseau et al., 2006c; Monsellier and Chiti, 2007; de Groot and Ventura, 2010). Whereas aggregation nucleating sequence segments can generally not be eliminated altogether without affecting the hydrophobic core of globular domains (Linding et al., 2004), their aggregation propensity can be minimized by placing charged residues like arginine $(\mathrm{R})$, lysine $(\mathrm{K})$, 
aspartate (D), glutamate (E) and also proline (P) directly at the flanks of these aggregation-prone sequences (Rousseau et al., 2006b). These so-called aggregation gatekeepers suppress aggregation by the repulsive effect of their charge (R, $\mathrm{K}, \mathrm{D}, \mathrm{E}$ ), the entropic penalty on aggregation due to their large and flexible side-chains (R, K) and the incompatibility with the beta-structure of aggregates due to the absence of a hydrogen carbon in its main chain $(\mathrm{P})$. At the same time their presence at the flanks of aggregating segments results in their structural positioning at exposed positions, often loops, thereby leaving the hydrophobic core unharmed.

Not surprisingly, chaperones and other members of the protein quality control system largely bind aggregationprone, hydrophobic sequences. Evidence also suggests that flanking gatekeepers might in some way contribute to or at least facilitate chaperone substrate recognition, suggesting the possibility of co-evolution between chaperone-binding specificities and gatekeeper residues (Rudiger et al., 1997, 2001; Patzelt et al., 2001; Rousseau et al., 2006b). In order to investigate whether gatekeepers can modulate the proteostatic regulation of an aggregating sequence and affect bacterial fitness, we have constructed a library composed of the amino-terminal fusion of a short aggregating segment from Escherichia coli protein RNA polymerase Sigma-32 factor (P-VLYLQ-D) flanked with different gatekeepers and nongatekeepers with the reporter protein EGFP.

Here we show that gatekeepers affect bacterial fitness of $\sigma 32 \beta-$ GFP overexpressing MC4100 E.coli cells and that gatekeepers modify the proteostatic regulation of $\sigma 32 \beta-$ GFP, affecting synthesis and degradation rates, expression levels and inclusion body formation. Furthermore, we provide evidence that could help explain the selective enrichment of gatekeeper residues flanking aggregating sequences in the E.coli proteome.

\section{Materials and methods}

\section{Plasmid construction}

The bacterial expression plasmid $\mathrm{pBAD} / \mathrm{Myc}$-His A (Invitrogen) was used for the construction of all the Sigma constructs. The Sigma wild-type sequence (P-VLYLQ-D) was fused to the amino-terminus of the reporter protein EGFP via a linker. Four amino-terminal residues were added to the construct to take into account the amino-terminal degradation rule. The native gatekeepers in the Sigma wild-type sequence (proline on the amino-terminus and aspartate at the carboxyterminus) were systematically mutated at the gatekeeper positions with arginine, lysine, aspartate, glutamate, proline, serine, alanine, glycine, valine and glutamine, while keeping the aggregating nucleating region (VLYLQ) constant.

\section{Bacterial strains and transformation}

The bacterial strains used in this study are as follows: Escherichia coli MC4100 Genotype: F-araD139 $\delta(\arg \mathrm{F}-$ lac)U169 rpsL150 (Str $\left.{ }^{\mathrm{r}}\right)$ relA1 flbB3501 deoC1 ptsF25 rbsR, Escherichia coli MC4100 $\Delta$ dnaK Genotype: $\Delta$ dnaK52: Cm sidB1 (BB1553), Escherichia coli MC4100 $\Delta$ TF Genotype: $\Delta$ tig : Kan (BB6515).

The cell lines were transformed with the construct of interest and kept on ice for $30 \mathrm{~min}$, allowing the entrance of the foreign DNA in the cell. After a 40 -s heat shock at $42^{\circ} \mathrm{C}$, the samples were kept on ice for 2 min. Next, Luria Bertani (LB) medium was added and the samples were incubated at $37^{\circ} \mathrm{C}$ for $1 \mathrm{~h}$. Subsequently, the samples were grown overnight at $37^{\circ} \mathrm{C}$ on agar plates that contained the selective marker ampicillin $(50 \mu \mathrm{g} / \mathrm{ml})$. By picking a single colony from the plate with the construct of interest, overnight cultures were grown in LB medium containing ampicillin $(50 \mu \mathrm{g} / \mathrm{ml})$ at $37^{\circ} \mathrm{C}$. Next, the overnight cultures were diluted and grown until an OD 0.6 was reached. The expression of the constructs was induced by adding L-arabinose $(0.02 \%)$ for $3 \mathrm{~h}$ unless stated otherwise.

\section{Determination of the in vivo solubility}

The wells of a 96-well plate (Cellstar ${ }^{\circledR}$ 96-well microplates TC, Greiner Bio-One) were filled with LB medium containing ampicillin $(50 \mu \mathrm{g} / \mathrm{ml})$ and inoculated with the right colony. The plates were sealed with a breathable film (BF-400, Lucron Bioproducts BVBA) and incubated overnight at $37^{\circ} \mathrm{C}$ at $120 \mathrm{rpm}$ in a shaker (Innova ${ }^{\circledR}-44$, New Brunswick Scientific). The next day, the cultures were diluted into a new plate and grown until they reached OD 0.6. The expression of the constructs was induced by adding L-arabinose (final concentration $0.02 \%$ ). After overnight incubation at $37^{\circ} \mathrm{C}$ at $120 \mathrm{rpm}$, the GFP fluorescence and OD signal of the plates were measured in a platereader (FLUOstar OPTIMA BMG $\mathrm{LABTECH})$. The $\mathrm{OD}$ was measured at $25^{\circ} \mathrm{C}$ at $600 \mathrm{~nm}$ ( 1 cycle, 20 flashes per well and per cycle). The GFP fluorescence was measured at $25^{\circ} \mathrm{C}$ with excitation at $485 \mathrm{~nm}$ and emission at $520 \mathrm{~nm}$ ( 1 cycle, 20 flashes per well and per cycle, well scanning matrix).

\section{Electrophoresis and western blot}

All samples were separated by sodium dodecyl sulphatepolyacrylamide gel electrophoresis (SDS-PAGE) (NuPAGE system, Invitrogen, USA). The SDS-PAGE was performed with NuPAGE Novex 10\% Bis-Tris precast gels (Invitrogen) and MES buffer (50 mM MES pH 7.2; $50 \mathrm{mM}$ Tris Base; $0.1 \%$ SDS; $1 \mathrm{mM}$ EDTA) under fixed voltage $(200 \mathrm{~V})$ for $35 \mathrm{~min}$ at room temperature. The gels were transferred to a nitrocellulose membrane surrounded by Whatman papers that were soaked into Towbin blot transfer buffer $(25 \mathrm{mM}$ Tris Base; $192 \mathrm{mM}$ glycine; 20\% MeOH; 0.1\% SDS). The transfer was performed in a Trans-Blot SD Cell (BioRad) under fixed current $(0.4 \mathrm{~A})$ for $30 \mathrm{~min}$ at room temperature. The protein transfer on the membrane was verified by PonceauS staining. After destaining the PonceauS from the membrane, it was blocked overnight at $4{ }^{\circ} \mathrm{C}$ in $5 \%$ skim milk powder in TBST $\quad(20 \mathrm{mM}$ Tris $\mathrm{pH} \quad 7.5 ; 150 \mathrm{mM} \quad \mathrm{NaCl} ; \quad 0.05 \%$ Tween-20). To detect the GFP-tagged Sigma constructs on the membrane, the primary antibody (anti-GFP, rabbit polyclonal) was diluted 1:10000 in TBST containing 5\% milk powder and incubated for $1 \mathrm{~h}$ at room temperature. After three times of rinsing with TBST, the membrane was incubated with a 1:5000 dilution of horseradish peroxidaseconjugated anti-rabbit IgG (Promega, USA) in TBST for $30 \mathrm{~min}$ at room temperature. After three times rinsing with TBST, the membrane was visualized with electrochemical luminescence (ECL) system (ChemiDoc XRS, BioRad).

\section{Synthesis assay}

After inducing the expression of the constructs, $1 \mathrm{ml}$ of the culture was collected at given time points $(0-10-20-40-$ 

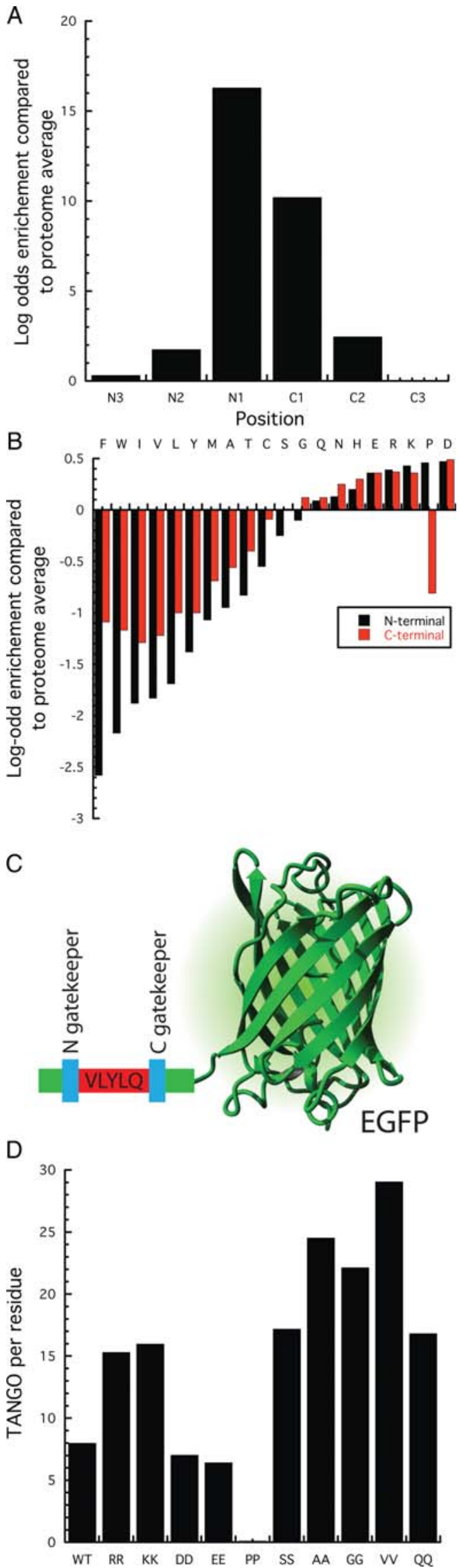

Fig. I. Gatekeeper placement in the E.coli proteome and the model construct. (A) Enrichment of gatekeeper residues (D, E, K, R and P) versus the proteome average at the first three amino- and carboxy-terminal
60-90-180 $\mathrm{min}$ ), and brought to $1 \mathrm{ml}$ of OD 0.6. After centrifugation at $5000 \mathrm{~g}$ during $10 \mathrm{~min}$, the pellets were lysed in $300 \mu \mathrm{l}$ buffer $(50 \mathrm{mM}$ Tris $\mathrm{pH} 7.5 ; 5 \mathrm{mM}$ EDTA; $150 \mathrm{mM}$ $\mathrm{NaCl} ; 1 / 25$ complete EDTA-free protease inhibitor cocktail tablets (Roche); 1/1000 kanamycin). The sample (14 $\mu 1)$ was denaturated for $5 \mathrm{~min}$ in the presence of $5 \% \mathrm{SDS}$ prior to SDS-PAGE (NuPAGE system, Invitrogen). Next, the gels were transferred to a nitrocellulose membrane and the membrane was probed with primary and secondary antibodies prior to visualization with ECL system (ChemiDoc XRS, BioRad). The details are described in the section 'Sodium dodecyl sulfate-polyacrylamide gel electrophoresis and western blot'.

\section{Degradation assay}

After a 3-h expression of the constructs, the culturing medium was changed into LB medium that contained ampicilin $(50 \mu \mathrm{g} / \mathrm{ml})$, glucose $(0.2 \%)$, repressing transcriptional activation and chloramphenicol $(200 \mu \mathrm{g} / \mathrm{ml})$, inhibiting bacterial protein synthesis. At given time points $(0-10-20-40-$ 60-90-180 $\mathrm{min}$ ), $1 \mathrm{ml}$ of the culture was collected and brought to $1 \mathrm{ml}$ of OD 0.6. After centrifugation at $5000 \mathrm{~g}$ during $10 \mathrm{~min}$, the pellets lysed in $300 \mu \mathrm{l}$ buffer $(50 \mathrm{mM}$ Tris $\mathrm{pH} 7.5 ; 5 \mathrm{mM}$ EDTA; $150 \mathrm{mM} \mathrm{NaCl}$; $1 / 25$ complete EDTA-free protease inhibitor cocktail tablets (Roche); $1 / 1000$ kanamycin). This sample represents the whole-cell fraction. The remaining lysate was further fractionated by centrifuging the sample at $15000 \mathrm{~g}$ for $5 \mathrm{~min}$ at $4^{\circ} \mathrm{C}$. The supernatant represents the soluble fraction. The pellet was resuspended in $200 \mu \mathrm{l}$ guanidine hydrochloride $(\mathrm{GdmHCl}$ $6 \mathrm{M}$ ) and subjected to a trichloroacetic acid precipitation. This sample represents the insoluble fraction. All samples $(14 \mu \mathrm{l})$ were denaturated for $5 \mathrm{~min}$ in the presence of $5 \%$ SDS prior to SDS-PAGE (NuPAGE system, Invitrogen). Next, the gels were transferred to a nitrocellulose membrane and the membrane was probed with primary and secondary antibodies prior to visualization with ECL system (ChemiDoc XRS, BioRad). The details are described in the section 'Sodium dodecyl sulfate-polyacrylamide gel electrophoresis and western blot'.

\section{Reverse transcription quantitative $P C R$}

After $3 \mathrm{~h}$ of expression, the transcriptional activity of different chaperones and proteases was determined by reverse transcription quantitative PCR (RT-qPCR).

Since bacterial mRNA has a very short half-life and genes can be induced during handling and processing of bacterial

positions flanking an aggregating sequence. The first position before and after an aggregating zone is strongly enriched in gatekeepers. By the third position the preference for gatekeeper residues vanishes and the proteome average is reached again. (B) Amino acid identity at the first three aminoand carboxy-terminal positions flanking an aggregating sequence. Proline is enriched only at the amino-terminal position. (C) Representation of the $\sigma 32 \beta-E G F P$ fusion construct. The short aggregating sequence $\sigma 32 \beta$ (VLYLQ, red) of the bacterial RNA polymerase factor $\sigma 32$ is fused to the amino-terminus of EGFP (green). The amino- and carboxy-terminal flanks (blue) of the aggregating sequence are systematically mutated as indicated in Table I, while keeping the aggregation-nucleating region constant. To make sure that all constructs have an identical sensitivity to the amino-terminal degradation rule, four amino acids (AMAM) are added before the amino-terminal gatekeeper. (D) The intrinsic aggregation propensities of the different $\sigma 32 \beta$-EGFP fusion constructs according to the TANGO algorithm. 
cells, the RNA was stabilized in vivo by adding the RNAprotect Bacteria Reagent (Qiagen) prior to lysis, allowing enough time for the efficient disruption of cells without the risk of distorting the gene expression profile. After enzymatic digestion of the bacterial cell wall by lysozyme (1 $\mathrm{mg} / \mathrm{ml}$ ), the total RNA was isolated using Rneasy Mini Kit (Qiagen, Germany). Reverse transcription was performed using the QuantiTect Rev. Transcription Kit (Qiagen) according to the manufacturer's instructions.

Quantitative real-time PCR was performed using TaqMan ${ }^{\circledR}$ Fast Universal PCR Master Mix $(2 \times)$ No AmpErase ${ }^{\circledR}$ UNG (Life Technologies ${ }^{\mathrm{TM}}$, USA) on an iCycler My-iQ Single Color Real-Time PCR detection system (Bio-Rad). Primers were developed with the RealTime PCR Assay Design Tool (Integrated DNA Technologies, Inc). The expression of each gene was normalized against gyrA, recA and zwf.

\section{Fluorescence microscopy}

After $3 \mathrm{~h}$ of expression, the bacterial sample was dried on a microscope slide prior to heat fixing. Antifade reagent (ProLong Gold, Invitrogen) was added and samples were kept in the dark for $24 \mathrm{~h}$. Images were acquired with a $\times 100$ oil objective of a fluorescence microscope (ECLIPSE TE2000, Nikon, Japan).

\section{Clonal selection}

Two constructs of interest were grown together in LB medium and ampicillin $(50 \mu \mathrm{g} / \mathrm{ml})$ by picking a single colony from the agar plate of each construct. The overnight cultures were diluted in a $50 \mathrm{ml}$ falcon tube containing fresh LB medium and ampicillin $(50 \mu \mathrm{g} / \mathrm{ml})$ and grown until they reached an OD of 0.6. The expression of the constructs was induced by adding L-arabinose $(0.02 \%)$. After $6 \mathrm{~h}$ of expression, the DNA was purified using the Miniprep KIT QIA prep (Qiagen) and sent for sequencing (VIB Genetic Service Facility). By investigating the chromatograms of the sent samples, the dominant construct could be assigned.

\section{Results}

\section{Charged residues are enriched at the flanks of aggregating sequences of the E.coli proteome}

We employed the TANGO algorithm (Fernandez-Escamilla et al., 2004) to identify all aggregation nucleating regions in the E.coli proteome. We then proceeded to analyze the frequency of each amino acid at the three positions aminoterminal as well as the three positions C-terminal of the aggregating sequence. It has been shown previously that charged residues (E, D, K and $\mathrm{R}$ ) and also $\mathrm{P}$ are significantly enriched at positions directly flanking aggregation nucleating regions (Rousseau et al., 2006b; Reumers et al., 2009a) as these residues oppose aggregation by charge repulsion and/or $\beta$-structure disruption (Silow and Oliveberg, 1997; Richardson and Richardson, 2002) and are therefore named aggregation gatekeepers. Fig. 1A shows the log-odds ratio of finding a gatekeeper residue in the indicated position compared with the proteome average of E.coli. It is clear that the first positions $\mathrm{N}$ - and $\mathrm{C}$-terminal of the aggregating regions are most strongly enriched in gatekeeper residues. This signal is very position specific: by the third position, the observed enrichment vanishes and the proteome average is again reached. There is a stronger enrichment on the $\mathrm{N}$-terminal position, which can be almost entirely ascribed to the stereochemistry of proline, which is enriched as a gatekeeper residue only at on the N-terminal position (Fig. 1B).

\section{Modulating gatekeeper identity of the $\sigma 32 \beta-E G F P$ fusion construct}

In order to probe the effect of gatekeeper residues on protein aggregation in E.coli, we have constructed an N-terminal fusion to GFP of an aggregating segment from the E.coli protein RNA polymerase factor sigma-32 (termed $\sigma 32 \beta$ ) with the amino acid sequence P-VLYLQ-D (see Fig. 1C). This construct was cloned in a pBAD expression vector, which allows tight repression of expression via glucose and induction via arabinose. In this construct the aggregating region is not part of a globular fold and therefore remains fully accessible for interaction. As indicated (Table I) the native gatekeepers of $\sigma 32 \beta$ Pro on the N-terminus and Asp on the carboxy-terminus were systematically mutated, whilst keeping the aggregation-nucleating region constant. Four constant $\mathrm{N}$-terminal residues (AMAM) were added to the construct to exclude differences in turnover rate due to N-terminal degradation.

\section{Gatekeepers affect bacterial fitness}

In order to assess whether gatekeepers have an effect on aggregating sequences in vivo and could help explain the evolutionary enrichment of these amino acids at the flanks of aggregating regions in the E.coli proteome, we grew mixed bacterial cultures, so that bacteria carrying one type of construct compete in the same medium with bacteria containing another type of construct. Pairwise competitive growth was performed by co-inoculating a single colony from each transformant of interest, growing them together in LB medium and ampicillin $(50 \mu \mathrm{g} / \mathrm{ml})$. The overnight cultures were diluted in a $50 \mathrm{ml}$ falcon tube containing fresh LB medium and ampicillin $(50 \mu \mathrm{g} / \mathrm{ml})$ and grown until they reached an OD of 0,6 . Expression of the constructs was induced by L-arabinose $(0,02 \%)$. After $6 \mathrm{~h}$ of expression, the DNA was purified and sequenced to establish the plasmid content of the mixed culture. In this way we were able to assess the effect of the gatekeeper residues on the bacterial fitness in a simple manner. The results from these experiments are shown in Table II (each condition was independently repeated 10 times). Interestingly, for all pair-wise

Table I. An overview of the gatekeeper constructs based on the wild-type $\sigma 32 \beta$ sequence. The central aggregating region is kept constant while the flanking residues are systematically changed

\begin{tabular}{llll}
\hline Name & N gatekeeper & C gatekeeper & Sequence \\
\hline wt & P & D & P-VLYLQ-D \\
RR & R & R & R-VLYLQ-R \\
KK & K & K & K-VLYLQ-K \\
DD & D & D & D-VLYLQ-D \\
EE & E & E & E-VLYLQ-E \\
PP & P & P & P-VLYLQ-P \\
SS & S & S & S-VLYLQ-S \\
AA & A & A & A-VLYLQ-A \\
GG & G & G & G-VLYLQ-G \\
VV & V & V & V-VLYLQ-V \\
QQ & Q & Q & Q-VLYLQ-Q \\
\hline
\end{tabular}


Table II. Results from pairwise competitive bacterial growth experiment. For this experiment cultures were simultaneously co-innoculated with two independent bacterial transformants containing different gatekeeper constructs. After a period of competitive growth, the plasmid content of the resulting culture was determined using classical Sanger sequencing. We always only found a single construct back (in 10 independent repeats), which we termed the dominant construct. The table shows the constructs that dominate for each pairwise growth competition performed

\begin{tabular}{llllllllllll}
\hline & WT & RR & KK & DD & EE & PP & SS & AA & GG & VV & QQ \\
\hline WT & & WT & WT & WT & WT & WT & WT & WT & WT & WT & WT \\
QQ & WT & RR & KK & DD & EE & PP & SS & AA & GG & VV & \\
VV & WT & RR & KK & DD & EE & PP & SS & AA & GG & & VV \\
& & & & & & & & & & & \\
KK & & & RR & DD & EE & & & & & & \\
RR & & & & DD & EE & & & & & & \\
DD & & & & & EE & & & & & & \\
\hline
\end{tabular}

combinations, the Sanger sequencing only turned up a single surviving construct, which we will call the dominant construct in what follows. We observed that the wild-type gatekeeper configuration (N-flanking Pro, C-flanking D) is dominant over all other flanking configurations. Second, non-wild-type charged gatekeeper flanks (EE,DD,RR,KK) are dominant over double proline flanks (PP) and nongatekeeped flanks (SS,AA,GG,QQ). Finally, we observe clear preferences between gatekeeper identities at the flanks of $\sigma 32 \beta$ ( $\mathrm{EE}>\mathrm{DD}>\mathrm{RR}>\mathrm{KK}$ ). These differences in bacterial fitness suggest that gatekeeper identity at the flanks of aggregating sequences have an influence on the manner in which aggregating sequences are handled by the cell.

\section{Gatekeepers affect protein expression by modulating protein synthesis and degradation rates}

In order to determine protein expression levels we measured the total GFP fluorescence of E.coli MC4100 cells after overnight expression of the different gatekeeper constructs (shown in Fig. 2A) The fluorescence intensity observed for the wild-type $\sigma 32 \beta$-GFP fusion is about half the intensity of GFP alone, and the constructs containing Arg, Lys, Asp or Glu as gatekeepers give similar fluorescence intensity as the wild-type fusion. However, without charged gatekeeper residues the fluorescence drops significantly. A decrease in total fluorescence generally indicates lower expression levels; however, aggregation can also result in the quenching of GFP fluorescence (Wurth et al., 2002). To address the aggregation of the different constructs we have observed bacterial cells by confocal fluorescence microscopy (Fig. 2B) and have quantified the percentage of cells with accumulation of GFP fluorescence in inclusions compared with diffuse distribution throughout the cells (Fig. 2C). This analysis reveals there is little or no inclusion formation of the wild-type $\sigma 32 \beta-$ GFP and charge-flanked $\sigma 32 \beta$-GFP fusions, suggesting that the observed decrease in fluorescence versus GFP alone directly results from a decreased expression due to the $\sigma 32 \beta$ fusion. On the other hand, the little fluorescence observable in the non-gatekeeped $\sigma 32 \beta-$ GFP fusions was largely found in inclusions (Fig. 2B and C). This suggests that the additional drop in fluorescence noticed in the absence of charged flanks could be caused by an additional drop in expression levels and/or by quenching due to increased aggregation and inclusion body formation. GFP quatification by western blot after $3 \mathrm{~h}$ of expression in E.coli MC4100 (Fig. 2D) reveals that both scenarios occur, depending on the identity of the residues substituting for the gatekeepers. On the one hand, Pro-, Ser- and Gln-flanked $\sigma 32 \beta-$ GFP are not detectable in the whole-cell lysate by western blot after $3 \mathrm{~h}$ of expression-although a minimal amount can be purified from inclusion bodies after $24 \mathrm{~h}$ of expression (data not shown)-showing that the decrease in signal is here mainly due to lower protein expression. On the other hand, Val- and Ala-flanked $\sigma 32 \beta-$ GFP are expressed at levels similar to wild-type- and gatekeeper-flanked $\sigma 32 \beta-\mathrm{GFP}$, suggesting that inclusion body formation is responsible here for the decreased fluorescence. Together these data show that the identity of the flanking residues affects both protein expression levels and aggregation, and that the presence of charged gatekeepers maximizes $\sigma 32 \beta-$ GFP expression while avoiding inclusion body formation.

To understand these differences in expression levels we measured $\sigma 32 \beta$-GFP synthesis and degradation rates in E.coli MC4100 (Fig. 3). The synthesis rate of the construct containing $\mathrm{Val}$ at the flanks is the highest, followed by the gatekeeper constructs containing Asp, Glu, Lys and Arg. The synthesis rate of the Ala-flanked construct is comparable to that of the Arg construct, and wild-type $\sigma 32 \beta-$ GFP experiences the lowest synthesis rate. Finally, the constructs containing Pro, Ser, Gly and Gln are hardly expressed so that no significant synthesis rate could be calculated (Fig. 3A). The effect of gatekeepers on the degradation rate of the $\sigma 32 \beta-$ GFP in E.coli MC4100 cells was investigated both in the soluble and insoluble fractions (Fig. 3B and C). $\sigma 32 \beta-$ GFP in the soluble fraction is stable over the $3 \mathrm{~h}$ of the experiment and no significant degradation could be observed for any $\sigma 32 \beta-$ GFP construct. A different scenario occurs in the insoluble fraction: the degradation rate of the constructs containing the gatekeeper Lys is the highest, followed by a similar degradation rate for the gatekeepers Arg, Asp and Glu, while non-gatekeeped constructs containing Val or Ala flanks are not degraded from the insoluble fraction.

Together these data demonstrate an important modulating role for the flanks of $\sigma 32 \beta$ on the expression, inclusion body formation, synthesis and degradation rates of $\sigma 32 \beta-\mathrm{GFP}$. Clearly in the case of $\sigma 32 \beta-$ GFP, charged gatekeeper residues favor fast protein synthesis and degradation, while avoiding inclusion body formation by efficiently degrading excess $\sigma 32 \beta-$ GFP from the insoluble fraction. This results in high overall expression yields of soluble $\sigma 32 \beta-$ GFP. Alaand Val-flanked $\sigma 32 \beta-$ GFP also achieve fast synthesis rates. However, in this case degradation of insoluble protein is much less efficient resulting in the accumulation of $\sigma 32 \beta-$ GFP in inclusion bodies. Pro-, Gly-, Ser- and Gln-flanked $\sigma 32 \beta-$ GFP show very inefficient protein synthesis. Moreover, the little amount of protein that gets expressed accumulates in inclusion bodies. Finally, wild-type $\sigma 32 \beta-$ GFP displays the most balanced behavior resulting in the best growth fitness: it has a slow expression rate but achieves expression yields that are as high as Asp-, Glu-, Arg- and Lys-flanked $\sigma 32 \beta-$ GFP. As a result, wild-type $\sigma 32 \beta-$ GFP expression does not produce insoluble protein and therefore avoids having to degrade misfolded protein from the insoluble fraction. 
A

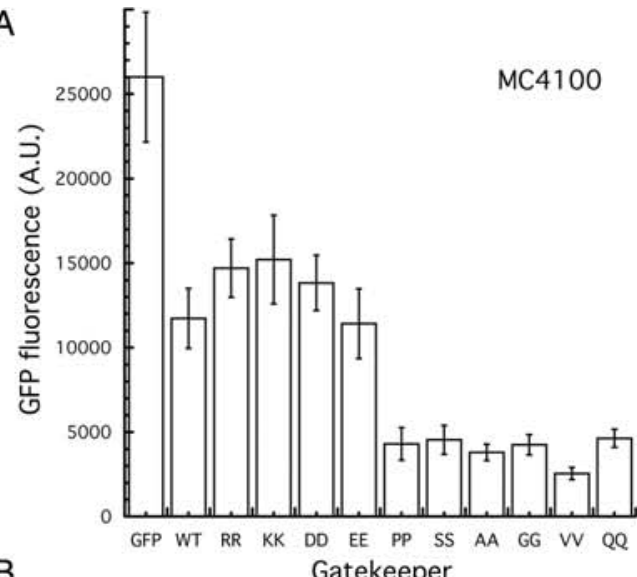

B
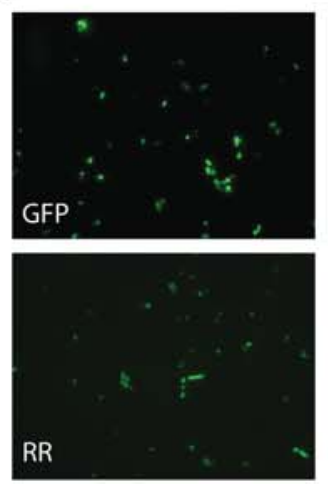

WT

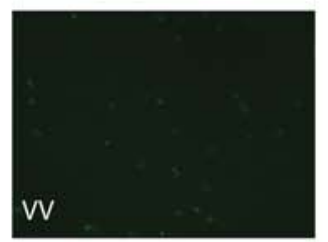

DD

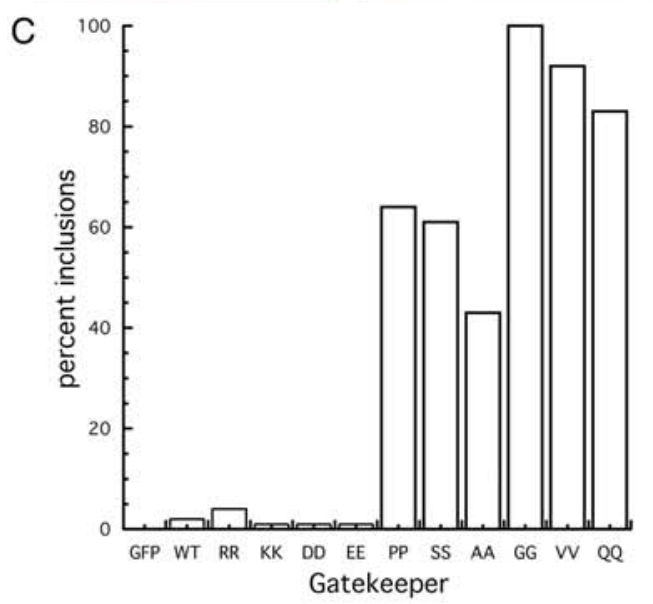

D

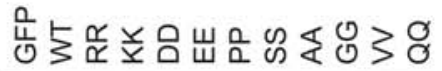

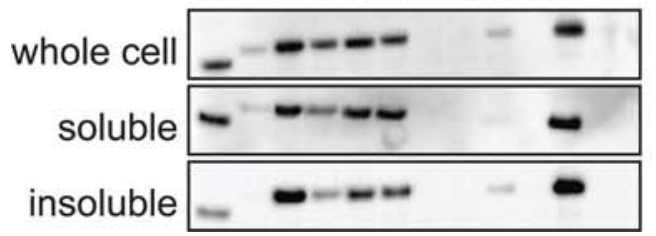

Fig. 2. Solutibility of gatekeeper variants upon expression of $\sigma 32 \beta-E G F P$ fusion constructs in E.coli MC4100 cells. (A) The GFP fluorescence signal after overnight expression. (B) Fluorescence microscopy of selected $\sigma 32 \beta-$ EGFP fusion constructs after $3 \mathrm{~h}$ of expression in E.coli MC4100 cells
Gatekeepers affect the proteostatic activity of MC4I 00 cells

Protein homeostasis (or proteostasis) is regulated both by transcriptional and post-translational mechanisms. It has been estimated that $\sim 40 \%$ of the variation in protein expression within a proteome can be explained by differences in mRNA levels (Vogel and Marcotte, 2012). The remaining variability is a result of co- and post-translational regulation. The protein quality control (PQC) machinery, consisting of ribosomes, chaperones and proteases, plays a central role in proteostasis.

In order to address the role of transcription for the regulation of $\sigma 32 \beta-$ GFP expression, we determined GFP mRNA levels by qPCR. Although some variation in mRNA levels can be observed (Fig. 4A), these do not correlate with the difference in protein expression observed for the different $\sigma 32 \beta$-GFP flanks, nor do they correlate with the bacterial growth (data not shown).

To determine whether gatekeeper identity is affecting proteostatic response by chaperones and proteases we also monitored chaperone and protease response by qPCR after $3 \mathrm{~h}$ of $\sigma 32 \beta-$ GFP expression. In total we monitored eight chaperone and proteolytic components of the PQC (Fig. 4B-D). In comparison to the proteostatic profile of $\sigma 32 \beta-$ GFP, charged gatekeeped flanks upregulate proteolytic components. All charged flanks display transcriptional upregulation of ClpP, and in addition, Lys, Asp and Glu flanks also have higher levels of ClpS. At the same time, chaperone mRNA levels are somewhat downregulated, especially DnaK and ClpB, suggesting a more efficient handling of these constructs by the chaperone machinery. Expression of $\sigma 32 \beta-$ GFP in the DnaK or trigger factor-defficient strains MC4100 $\Delta$ DnaK

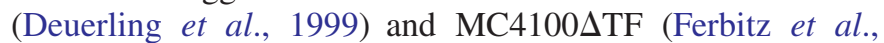
2004) confirms this, as knock out of DnaK or Trigger factor activity indeed strongly reduces $\sigma 32 \beta-$ GFP expression and $16 \mathrm{~S}$ rRNA levels in gatekeeped but not non-gatekeeped $\sigma 32 \beta-$ GFP-expressing cells (Fig. 5). Overall, these observations are consistent with the high expression levels and turnover rates observed for charge gatekeeped $\sigma 32 \beta-$ GFP. Non-gatekeeped $\sigma 32 \beta$ flanks display a more varied behavior. Gln-flanked $\sigma 32 \beta-$ GFP is expressed at low levels (Fig. 4A). At the same time it strongly upregulates chaperones DnaK and ClpB as well as the ClpP protease (Fig. 4D), suggesting acute toxicity of this construct. This is also suggested by the lowest pairwise growth fitness of this construct. On the other hand, Pro-, Ser- and Gly-flanked $\sigma 32 \beta-$ GFP are also barely expressed but they possess a PQC profile that is very similar to wild-type $\sigma 32 \beta$-GFP. However, both their 16S rRNA and their overall growth fitness are significantly lower than wildtype and charge gatekeeped $\sigma 32 \beta-$ GFP but still higher than Gln-flanked $\sigma 32 \beta-$ GFP (Fig. 5). This again suggests an increased toxicity of these constructs and/or the ability of surviving cells to downregulate $\sigma 32 \beta-$ GFP expression to non-toxic levels. Finally, the only two non-gatekeeped $\sigma 32 \beta-$ GFP that are significantly expressed also display very

showing a diffuse (wild type, RR, DD) or a punctate pattern (VV) or absence of fluorescence (QQ). (C) Quantification of the percentage of cells with accumulation of EGFP fluorescence in inclusions compared with the diffuse distribution throughout the cells. (D) Western blot analysis of the $\sigma 32 \beta$-EGFP fusion constructs after $3 \mathrm{~h}$ of expression in E.coli MC4100 cells in whole-cell lysates as well as soluble and insoluble fractions. 

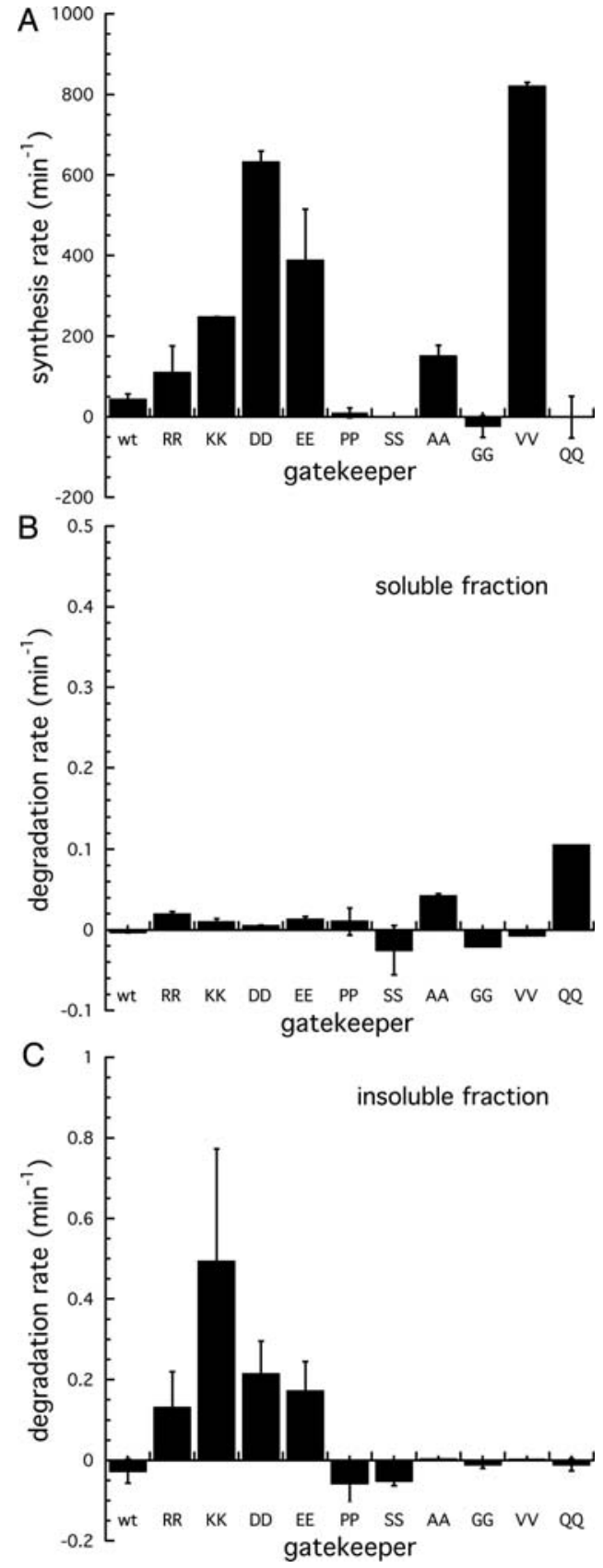

Fig. 3. Synthesis and degradation rates of gatekeeper variants. (A) Synthesis rate of the $\sigma 32 \beta$-EGFP fusion constructs when expressed in E.coli MC4100 cells determined in the first three hours of expression. (B) Degradation rate from the soluble fraction of all $\sigma 32 \beta$-EGFP fusion constructs in E.coli MC4100 cells. (C) Degradation rate of all $\sigma 32 \beta-$ EGFP fusion constructs from the insoluble fraction when expressed in E.coli MC4100 cells.

different behaviors. While Val-flanked $\sigma 32 \beta-$ GFP expresses at high levels-similar to the levels observed in wild-type and charged gatekeeped constructs-its PQC is downregulated in comparison to wild-type $\sigma 32 \beta-$ GFP. According to the aggregation prediction algorithm TANGO, this construct has the highest intrinsic aggregation propensity of all tested constructs (Fig. 1D), which is in accordance with its location in inclusion bodies and the inability of the MC4100 cells to degrade this protein from these inclusions. It is tempting to speculate that this very high aggregation propensity is the reason why MC4100 survive despite high $\sigma 32 \beta-\mathrm{GFP}$ expression. Indeed, it has been shown that increasing protein aggregation is an efficient manner to suppress aggregate toxicity, as aggregate toxicity generally results from smaller oligomeric aggregates (Balch et al., 2008; Lee et al., 2011; Eisenberg and Jucker, 2012; Laganowsky et al., 2012). The proteostatic response and expression level of Ala-flanked $\sigma 32 \beta-$ GFP are in agreement with that hypothesis. Whereas this construct is the second most aggregating $\sigma 32 \beta-$ GFP after VV, it is still expressed although at lower levels. Interestingly however, this lower expression is associated with the upregulation of the $\mathrm{ClpB}$ disaggregation-associated chaperone, which is another mechanism to avoid oligomer toxicity (Schlieker et al., 2004; Balch et al., 2008). Although the significant expression of these constructs clearly indicates their lack of toxicity, the overexpression of these constructs and their storage in inclusion bodies still comes at the price of decreased growth fitness. This is also confirmed by the $16 \mathrm{~S}$ rRNA levels of cells expressing Val-and Ala-flanked $\sigma 32 \beta-$ GFP: their $16 \mathrm{~S}$ rRNA levels are of the same magnitude as gatekeeped $\sigma 32 \beta-$ GFP but their competitive bacterial growth rate is significantly lower (Fig. 5A and Table II).

Together these data demonstrate that the residues directly flanking aggregation nucleating regions contain relevant information that can affect the manner in which the proteostatic machinery recognizes and handles exposed aggregation-prone sequences.

\section{Discussion}

Aggregation gatekeepers are enriched at the flanks of aggregation nucleating hydrophobic sequences, thereby increasing solubility and slowing aggregation kinetics (Rousseau et al., 2006c; Monsellier and Chiti, 2007; Monsellier et al., 2007; Reumers et al., 2009a; de Groot and Ventura, 2010). Here we investigated whether the identity of gatekeepers flanking an aggregation-prone sequence from the $\sigma 32$ transcription factor fused to GFP affects the manner in which E.coli responds to its expression in terms of growth fitness. Additionally, we investigated how differences in bacterial fitness relate to the proteostasis of the $\sigma 32-$ GFP protein, including expression levels, turnover rate, aggregation propensity and its impact on the transcriptional regulation of the protein quality control machinery. Importantly, we did not address growth fitness by comparing the absolute growth rate of monoclonal cultures, but set out to compare growth fitness by competitive growth of cultures incubated with equal amounts of two different $\sigma 32-$ GFP clones. These experiments revealed a significant difference in fitness induced by the expression of the different $\sigma 32-$ GFP constructs tested. Surprisingly expression of the $\sigma 32-\mathrm{GFP}$ fusion maintained highest fitness (P-VLYLQ-D), followed by charged gatekeeped $\sigma 32-\mathrm{GFP}$ (DD,EE,KK,RR), while the lowest competitive fitness was observed for non-charged flanks (PP,SS,GG,AA,VV,QQ), showing that the flanks of aggregation-prone sequences contribute to the manner in which E.coli MC4100 cells react to the expression of $\sigma 32-$ GFP. Overexpression of a recombinant protein represents a physiological stress that affects the growth of a bacterial culture by diverting a significant amount of metabolic energy from growth towards RNA transcription, protein translation and folding but also by the manner in which the expressed protein will be tolerated by the cell due, for instance, to aggregation and protein toxicity 

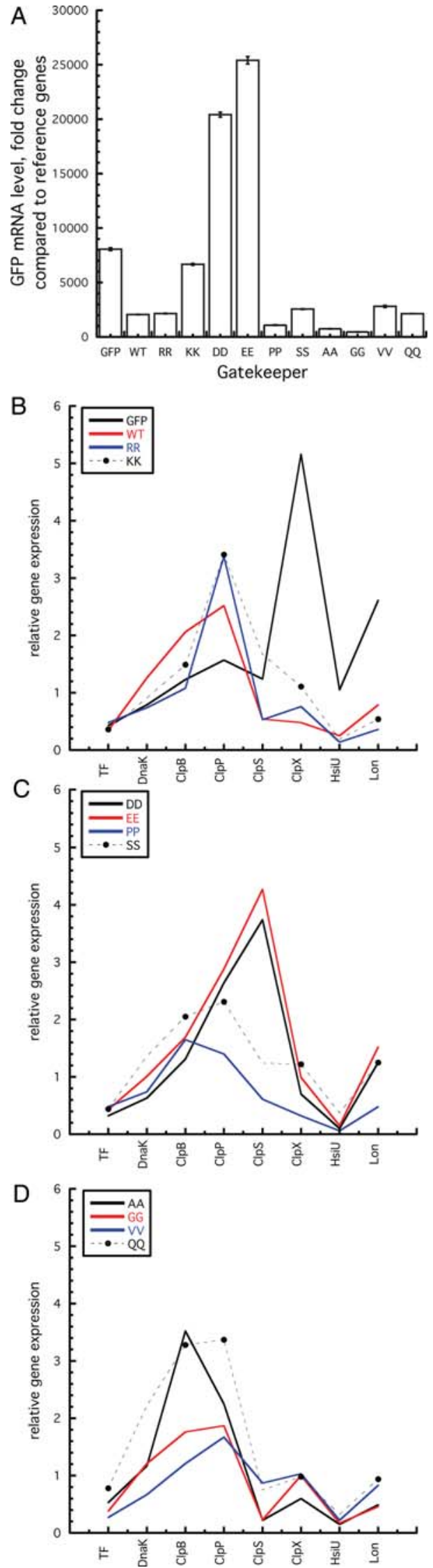

Fig. 4. Transcription levels (mRNA) of gatekeeper variants and of selected proteostatic components in E.coli MC4100 cells expressing gatekeeper variants. (A) GFP mRNA levels of $\sigma 32 \beta$-EGFP fusion constructs when expressed for $3 \mathrm{~h}$ in E.coli MC4100. (B) Chaperone and protease levels of
(Baneyx and Mujacic, 2004; Sabate et al., 2010; Hartl et al., 2011; Mogk et al., 2011). We did not observe an inverse relationship between bacterial fitness and protein expression levels. On the contrary, charge gatekeeped $\sigma 32 \beta$-GFP which display the highest competitive fitness also produce the highest levels of protein, while most non-gatekeeped $\sigma 32 \beta-$ GFP variants which have a lower fitness produce little protein. Neither do we observe a correlation between $\sigma 32 \beta-$ GFP mRNA levels and protein levels as positively charged gatekeepers have low mRNA levels in comparison to negatively charged gatekeepers while both result in high expression levels. These observations suggest that the differences in $\sigma 32 \beta$-GFP expression levels are mostly due to co- and post-translational events and that physiological stresses determining differences in bacterial fitness are due to qualitative differences in proteostatic response to the expression of $\sigma 32 \beta-$ GFP rather than to overexpression levels per se.

As protein aggregation is often toxic differences in bacterial fitness and $\sigma 32 \beta-$ GFP expression might originate from differences in intrinsic aggregation propensity of gatekeeped and non-gatekeeped $\sigma 32 \beta$. More specifically aggregate toxicity has been shown to originate from soluble oligomeric aggregates whereas larger insoluble aggregates are generally less toxic (Bucciantini et al., 2002; Eisenberg and Jucker, 2012). As a result, it has been shown that aggregate toxicity can be suppressed either by removing these smaller aggregates or on the contrary by recruiting these aggregates in larger inclusions (Cohen et al., 2006). Whereas this remains to be investigated in more detail, our data are in agreement with such a scenario. The wild-type and charge-flanked $\sigma 32 \beta$ have the lowest aggregation propensity implying a lower protein toxicity and accordingly have the highest growth fitness despite high expression levels. At the other extreme, the two most strongly aggregating non-gatekeeped $\sigma 32 \beta$ constructs (Val and Ala flanks) also express significant amounts of protein to levels that are comparable to wild-type and gatekeeped $\sigma 32 \beta-$ GFP. Although bacterial fitness is clearly affected by aggregation, proliferating cells survive while accumulating large amounts of protein in inclusion bodies. This is in contrast to non-gatekeeped $\sigma 32 \beta$-GFP constructs with intermediate aggregation propensity (such as Ser, Gln or Gly flanks) where growth fitness is equally affected but where proliferating cells survive by almost completely suppressing $\sigma 32 \beta$-GFP expression while accumulating the little protein produced in inclusion bodies.

As growth fitness and $\sigma 32 \beta-$ GFP expression therefore seem to be determined by the intrinsic aggregation propensity and toxicity of each $\sigma 32 \beta-$ GFP mutant, the observed changes in expression levels and inclusion body formation allowing bacterial survival clearly results from a shift in proteostatic regulation. Mutation at gatekeeper positions results in changes in synthesis and degradation rates, affects chaperone dependence and modulates the transcriptional regulation of chaperone and protease levels and it remains to be seen whether differences in $\sigma 32 \beta$-GFP mRNA levels are themselves not the result of some feedback mechanism coupling protein quality control to transcriptional control. Structural

$\sigma 32 \beta-$ EGFP fusion constructs when expressed for $3 \mathrm{~h}$ in E.coli MC4100 for GFP alone as well as the wild-type WT $\sigma 32 \beta$-EGFP sequence and RR and KK variants. (C) Equivalent data for DD, EE, PP and SS constructs. (D) Equivalent data for AA, GG, VV and QQ constructs. 

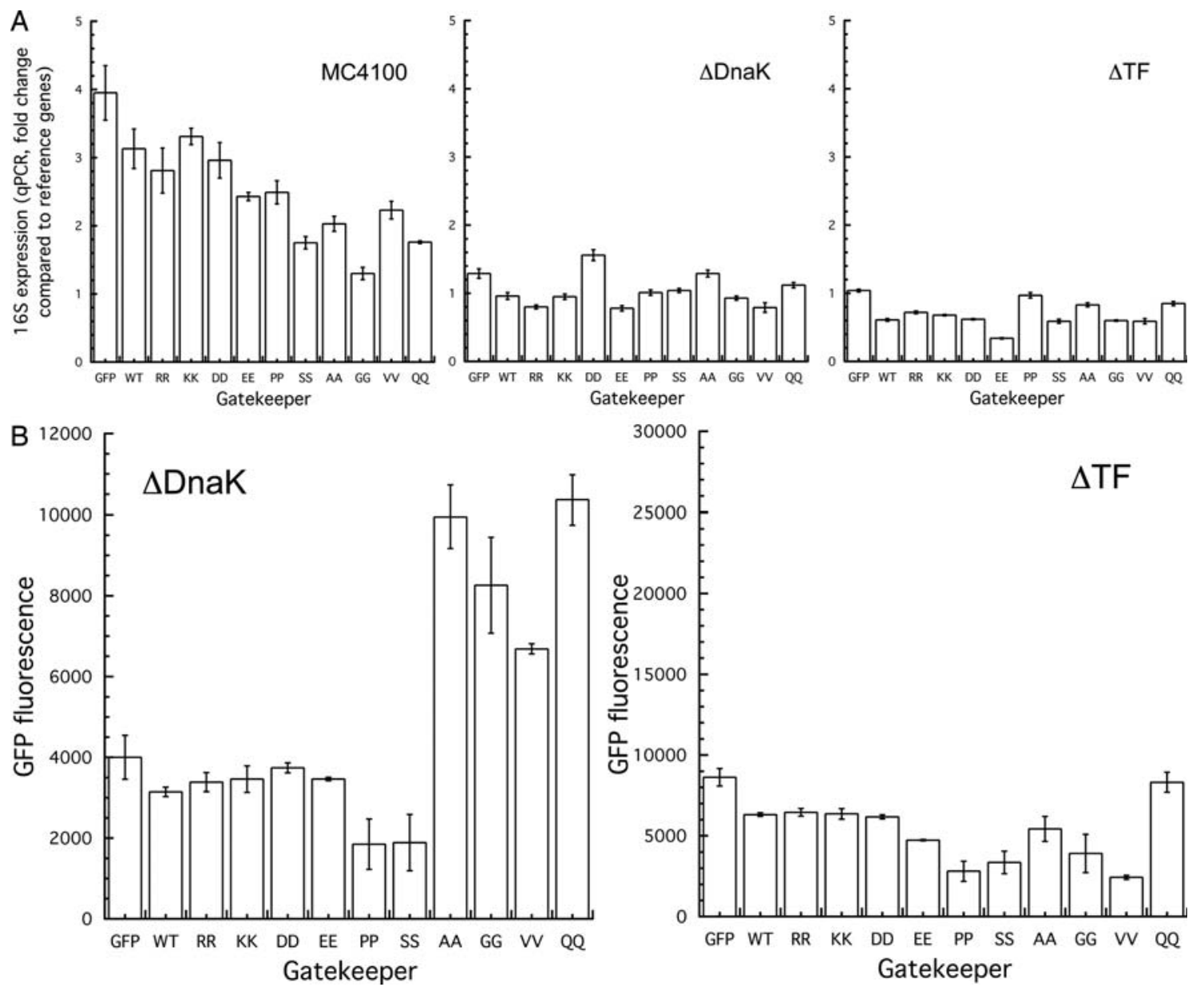

Fig. 5. Expression profiles in deletion strains for DnaK and Trigger Factor. (A) $16 \mathrm{~S}$ ribosomal RNA levels after expression of the $\sigma 32 \beta-E G F P$ fusion constructs in E.coli MC4100 wild type, $\Delta$ DnaK and $\Delta \mathrm{TF}$ cell lines. (B) GFP fluorescence signal of the $\sigma 32 \beta-$ EGFP fusion constructs after $3 \mathrm{~h}$ expression in E.coli $\mathrm{MC} 4100 \Delta \mathrm{DnaK}$ and $\Delta \mathrm{TF}$ cell lines.

and biophysical evidence suggest a molecular origin for the effect of charges on proteostatic regulation. The ribosomal tunnel for instance possesses a negative potential allowing for more frequent translational pausing with positive charges and faster conversion of negative and neutral sequences ( $\mathrm{Lu}$ and Deutsch, 2008). Chaperones also seem to favor (often positive) charges at their flanks (Rodriguez et al., 2008), although it remains unclear whether these charges directly contribute to binding (which is the case for DnaJ; Rudiger et al., 2001) or whether they facilitate capturing of a hydrophobic substrate sequence by charge repulsion (which is the lost likely scenario for DnaK; (Rudiger et al., 1997). Protease function and protein translocation is also highly dependent on charge, suggesting further possibilities for modulating protein homeostasis by charges. Flanking charges to aggregating sequences therefore seems an optimal strategy to couple proteostasis with the protein aggregation propensity of individual aggregating sequences.

While many points require a more in-depth inquiry, our data demonstrate a role for gatekeeper residues in modulating the proteostatic regulation of aggregating protein sequences allowing cells to survive high aggregation loads despite an evident cost in competitive fitness. Mutation at gatekeeper positions directly flanking aggregating sequences therefore suggests an evolutionary mechanism allowing the creation of variation in the proteostatic regulation of proteins without affecting their core structure, which is directly checked by selection on competitive growth fitness.

\section{Acknowledgements}

The wild-type bacterial strain MC4100 was kindly donated by Prof. D. Charlier (Vrije Universiteit Brussel) and the $\Delta \mathrm{TF}$ and $\Delta$ DnaK MC4100 strains by Bernd Bukau (ZMBH Heidelberg).

\section{Funding}

The VIB Switch Laboratory was supported by the Research Foundation Flanders (FWO), the Agency for Innovation by Science and Technology Flanders (IWT), the Federal Office for Scientific Affairs, Belgium IUAP P6/43 and the Flanders Institute for Biotechnology (VIB). J.X. was supported by Linking Sino-European Universities through Mobility and National Natural Science Foundation of China (81000861). F.D. was supported by a postdoc fellowship from the Biomedical Group of KULeuven.

\section{References}

Anfinsen,C.B. and Scheraga,H.A. (1975) Adv. Protein Chem., 29, 205-300. Balch,W.E., Morimoto,R.I., Dillin,A. and Kelly,J.W. (2008) Science, 319, 916-919.

Baneyx,F. and Mujacic,M. (2004) Nat. Biotechnol., 22, 1399-1408.

Bucciantini,M., Giannoni,E., Chiti,F., et al. (2002) Nature, 416, 507-511.

Chiti,F. and Dobson,C.M. (2006) Annu. Rev. Biochem., 75, 333-366. 
Cohen,E., Bieschke,J., Perciavalle,R.M., Kelly,J.W. and Dillin,A. (2006) Science, 313, 1604-1610.

De Baets,G., Reumers,J., Delgado Blanco,J., Dopazo,J., Schymkowitz,J. and Rousseau,F. (2011) PLoS Comput. Biol., 7, e1002090.

de Groot,N.S. and Ventura,S. (2010a) PLoS One, 5, 1-17.

De Simone,A., Dhulesia,A., Soldi,G., Vendruscolo,M., Hsu,S.T., Chiti,F. and Dobson,C.M. (2011) Proc. Natl Acad. Sci. USA, 108, 21057-21062.

Deuerling,E., Schulze-Specking,A., Tomoyasu,T., Mogk,A. and Bukau,B. (1999) Nature, 400, 693-696.

Dobson,C.M. (2001) Philos. Trans. R. Soc. Lond. B. Biol. Sci., 356, $133-145$.

Eisenberg,D. and Jucker,M. (2012) Cell, 148, 1188-1203.

Ferbitz,L., Maier,T., Patzelt,H., Bukau,B., Deuerling,E. and Ban,N. (2004) Nature, 431, 590-596.

Fernandez-Escamilla,A.M., Rousseau,F., Schymkowitz,J. and Serrano,L. (2004) Nat. Biotechnol., 22, 1302-1306.

Fitzpatrick,A.W., Knowles,T.P., Waudby,C.A., Vendruscolo,M. and Dobson,C.M. (2011) PLoS Comput. Biol., 7, e1002169.

Goldschmidt,L., Teng,P.K., Riek,R. and Eisenberg,D. (2010) Proc. Natl Acad. Sci.USA, 107, 3487-3492.

Guisbert,E., Herman,C., Lu,C.Z. and Gross,C.A. (2004) Genes Dev., 18, $2812-2821$

Hartl,F.U., Bracher,A. and Hayer-Hartl,M. (2011) Nature, 475, 324-332.

Laganowsky,A., Liu,C., Sawaya,M.R., et al. (2012) Science, 335, $1228-1231$

Lee,J., Culyba,E.K., Powers,E.T. and Kelly,J.W. (2011) Nat. Chem. Biol., 7, 602-609.

Linding,R., Schymkowitz,J., Rousseau,F., Diella,F. and Serrano,L. (2004) J. Mol. Biol., 342, 345-353.

Lu,J. and Deutsch,C. (2008) J. Mol. Biol., 384, 73-86.

Mogk,A., Huber,D. and Bukau,B. (2011) Cold Spring Harbor Perspect. Biol., 3, a004366.

Mogk,A., Tomoyasu,T., Goloubinoff,P., Rudiger,S., Roder,D., Langen,H. and Bukau,B. (1999) Embo J., 18, 6934-6949.

Monsellier,E. and Chiti,F. (2007) EMBO Rep., 8, 737-742.

Monsellier,E., Ramazzotti,M., de Laureto,P.P., Tartaglia,G.G., Taddei,N., Fontana,A., Vendruscolo,M. and Chiti,F. (2007) Biophys. J., 93, $4382-4391$

Muchowski,P.J. and Wacker,J.L. (2005) Nat. Rev. Neurosci., 6, 11-22.

Patzelt,H., Rudiger,S., Brehmer,D., et al. (2001) Proc. Natl Acad. Sci. USA, 98, 14244-14249.

Reumers,J., Maurer-Stroh,S., Schymkowitz,J. and Rousseau,F. (2009a) Hum. Mutat., 30, 431-437.

Reumers,J., Rousseau,F. and Schymkowitz,J. (2009b) Open Biol., 2, $176-184$.

Richardson,J.S. and Richardson,D.C. (2002) Proc. Natl Acad. Sci. USA, 99, $2754-2759$.

Rodriguez,F., Arsene-Ploetze,F., Rist,W., Rudiger,S., Schneider-Mergener,J., Mayer,M.P. and Bukau,B. (2008) Mol. Cell, 32, 347-358.

Rousseau,F., Schymkowitz,J. and Serrano,L. (2006a) Curr. Opin. Struct. Biol., 16, 118-126.

Rousseau,F., Serrano,L. and Schymkowitz,J.W. (2006b) J. Mol. Biol., 355, $1037-1047$.

Rousseau,F., Serrano,L. and Schymkowitz,J.W.H. (2006c) J. Mol. Biology, 355, $1037-1047$.

Rudiger,S., Germeroth,L., SchneiderMergener,J. and Bukau,B. (1997) Embo J., 16, 1501-1507.

Rudiger,S., Schneider-Mergener,J. and Bukau,B. (2001) EMBO J., 20, $1042-1050$

Sabate,R., de Groot,N.S. and Ventura,S. (2010) Cell. Mol. Life Sci., 67, $2695-2715$.

Schlieker,C., Weibezahn,J., Patzelt,H., et al. (2004) Nat. Struct. Mol. Biol., 11, 607-615.

Silow,M. and Oliveberg,M. (1997) Proc. Natl Acad. Sci. USA, 94, 6084-6086.

Soti,C., Pal,C., Papp,B. and Csermely,P. (2005) Curr. Opin. Cell Biol., 17, 210-215.

Vogel,C. and Marcotte,E.M. (2012) Nat. Rev. Genet, 13, 227-232.

Wurth,C., Guimard,N.K. and Hecht,M.H. (2002) J. Mol. Biol., 319, $1279-1290$. 